\title{
On the Anomalous Deliquescence of Sea-Spray Aerosols
}

\author{
R. F. Pueschel, ${ }^{1}$ R. J. Charlson and N. C. Ahlquist \\ Dept. of Civil Engineering, University of Washingion, Seallle \\ 3 July 1969 and 2 September 1969
}

\section{Introduction}

Interest in the relationship in the atmosphere between cloud and fog droplets, raindrops, and salt particles dates back to the early measurements of hydrometeor salinities by Kohler. This interest is currently embodied in studies of the role of giant sea-salt particles in the formation of large droplets in clouds, and their influence in restricting visibility. In both cases, the size of the droplet to which hygroscopic particles can grow in environments of defined water contents is of utmost importance.

Experimental techniques for investigation of the hygroscopicity of salt particles in the past usually involved their direct microscopic observation in changing environmental humidities. The support of individual particles necessary for this type of investigation, such as spider webs, could have influence on the results. In situ measurements, on the other hand, are possible using light scattering techniques without interfering with the particles. The extinction coefficient $b$ due to scattering

\footnotetext{
${ }^{1}$ Present affiliation: ESSA Atmospheric Physics and Chemistry Laboratory, Boulder, Colo.
}

can be related to particle radius $r$ with the $b \propto r^{p}$ dependence. Since in most cases $p>1$, the detection of even small differences in size of the particles is possible. The integrating nephelometer (Ahlquist and Charlson, 1967) measures the light scattering coefficient $b$ at atmospheric levels, and has the added advantage of being a windowless and lensless device so that condensation on such components cannot interfere with the results.

The instrument has been applied to an investigation of the deliquescence of sea-spray aerosols. While a subsequent paper will report more on all details of this investigation, this note is intended to merely call attention to one particular finding: there exists an apparent difference in growth between salt particles produced by the sea and those produced from a pure sodium chloride solution when subjected to increasing humidity. This finding leads to the conclusions that pure $\mathrm{NaCl}$ crystal properties cannot be taken to represent a sea-spray crystal for theoretically or experimentally investigating the deliquescent behavior of such particles, that existing visibilities do not correspond to that deduced from the theoretical growth equation for a particular salt content 
and relative humidity, and that there are cases where droplet formation on giant sea-salt particles occurs to only a limited extent with the possible consequence of invalidating the sea-salt hypothesis.

\section{Apparatus and results}

The effect became apparent for the first time during a. field experiment at the coast of the Pacific ocean. A maritime aerosol was aspirated through the nephelometer, in front of which was a humidifier consisting of a $2.5-\mathrm{cm}$ diameter aluminum tube lined with felt and wrapped with a $0-500 \mathrm{~W}$ heating tape. Liquid water was fed into this tube at varying rates and vaporized to result in relative humidities $R_{T}$ in a range $30 \%<R_{T}$ $<100 \%$ inside the nephelometer. The relative humidity was measured with a dry-bulb wet-bulb psychrometer at the outlet of the nephelometer with adequate air velocity provided by the aspiration blower. The result was a drastic increase in light scattering at relative humidities approaching $70 \%$, but hardly any increase (i.e., inhibited droplet growth) at humidities $>80 \%$, in contradiction to expectations based on the thermodynamics of condensation.

The phenomenon was subsequently investigated in more detail in the laboratory utilizing a different

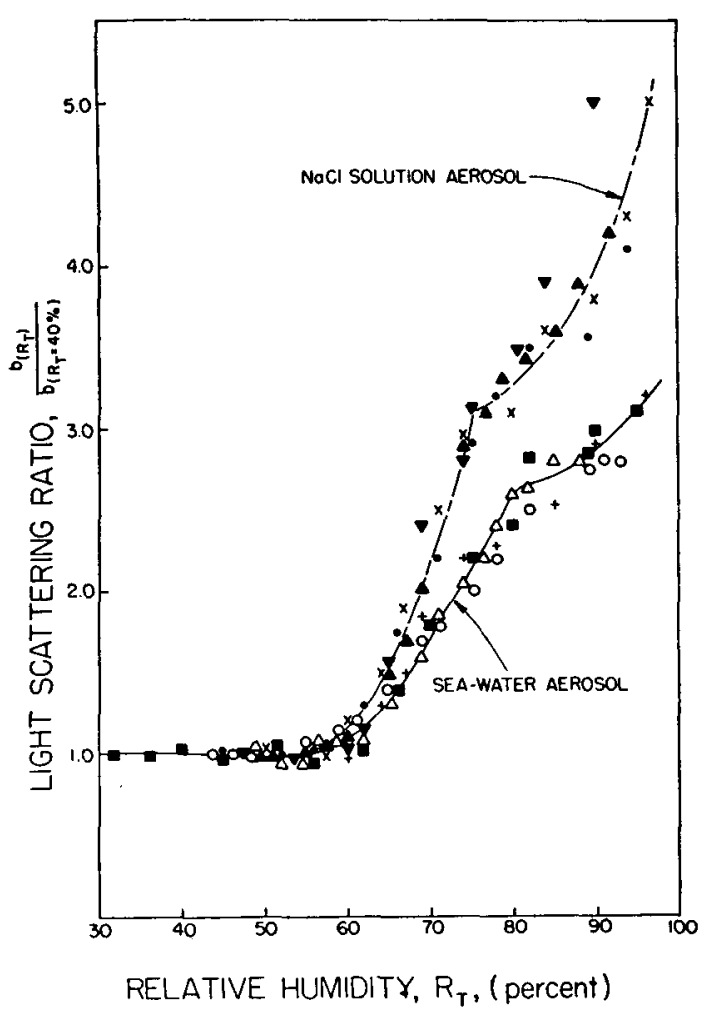

FIG. 1. Variation of light scattering ratio, $b\left(R_{T}\right) / b\left(R_{T}=40 \%\right)$ with relative humidity of the air for a $3 \% \mathrm{NaCl}$ solution aerosol and a sea-water aerosol. Legend: $X,+, \dot{R}_{T} \approx 50 \% \mathrm{sec}^{-1} ; \Delta, \triangle$, $\dot{R}_{T} \approx 1 \% \mathrm{~min}^{-1} ; \bullet, 0, \dot{R}_{T} \approx 0.1 \% \mathrm{~min}^{-1} ; \mathbf{\nabla}$, solution of $3 \% \mathrm{NaCl}$, $0.2 \% \mathrm{MgCl}_{2}$ and $0.1 \% \mathrm{MgSO}_{4} ; \mathrm{G}$, natural sea-spray aerosol. humidification apparatus. Salt aerosols were produced in a chamber of $20 \mathrm{~m}^{3}$ volume by flowing 20 liters $\mathrm{min}^{-1}$ of air through a fritted glass sparger submerged in either a $3 \%$ by weight sodium chloride solution, a $3 \% \mathrm{NaCl}$ solution to which $0.2 \% \mathrm{MgCl}_{2}$ and $0.1 \%$ $\mathrm{MgSO}_{4}$ were added, or a sample of natural sea water. The generator was operated until the aerosol concentration at low $\left(R_{T}<45 \%\right)$ humidity reached a known and reproducible value. The chamber was purged with filtered air prior to each run and the clean air scattering background chosen as zero point for the salt aerosol concentration. The relative humidity was then gradually increased inside the whole aerosol chamber by vaporizing known amounts of water, providing a continuous record of the aerosol light scattering coefficient as function of relative humidity. Fig. 1 is a summary of the responses of light scattering to relative humidity for the several different aerosols. The upper curve corresponds to the variations in the light scattering coefficient with humidity for a pure $\mathrm{NaCl}$ solution, i.e., a synthetic, inorganic, sea-water aerosol. Runs were made with several variations in the rate of humidification, viz., $\dot{R}_{T}=50 \% \mathrm{sec}^{-1}, \dot{R}_{T}=1 \% \mathrm{~min}^{-1}$, and $\dot{R}_{T}=0.1 \% \mathrm{~min}^{-1}$, where $\dot{R}_{T}$ is the time rate of change of relative humidity. The inorganic ion composition of the solute was varied by adding magnesium chloride and magnesium sulfate in proportions intended to more realistically represent the inorganic chemical composition of sea water, and the results do not deviate significantly from those for the pure $\mathrm{NaCl}$ solution. The lower curve, on the other hand, gives the variation of light scattering with humidity for laboratory-produced sea water aerosols. Again, various time rates of humidification identical to those above were employed. In addition, results from measurements on natural aerosols at the sea shore with a humidification rate $\dot{R}_{T}=50 \% \mathrm{sec}^{-1}$ are represented in the graph. Neither the sea-spray aerosol nor the results at different humidification rates deviate significantly from the general pattern of the curve which, however, is altogether different from the results from the pure $\mathrm{NaCl}$ solution.

In order to eliminate variations in the initial size of either crystals or droplets as a cause for the effect, corresponding size distributions were measured with a flame scintillation spectrophotometer (Pueschel, 1969) and are represented in Fig. 2, in which the size frequency distributions of the salt aerosol from the pure $\mathrm{NaCl}$ solution, the sea-water aerosol produced in the laboratory, and a natural sea-spray aerosol measured at the sea shore and produced by the processes of bursting bubbles and surface wave action by the ocean, are given. Of particular significance for the following discussion is the similarity of the aerosol size distributions produced in the laboratory from the sodium chloride solution and from the sea-water sample. The slight increase in smaller particles, and the slight shift in the distribution maximum for the sea-water sample is probably due to the lower surface tension of this sample compared to 
the pure salt solution. Since, however, only particles of size $r>0.1 \mu$ contribute significantly to light scattering, these deviations have no influence on the results.

\section{Discussion}

Some definite conclusions can be drawn from the results in Figs. 1 and 2. 1) The phenomenon is restricted to solution droplets, i.e., it is most clearly in evidence at humidities above that at which transition from crystal to droplet occurs. 2) From the results and from theory (Keith and Arons, 1954), it follows that for the particle sizes in question the effect is independent of time, at least for the time scales employed in this investigation, so that it may be discussed in terms of phase equilibria rather than growth rate kinetics. 3) Addition to the pure $\mathrm{NaCl}$ solution of $\mathrm{MgCl}$ and $\mathrm{MgSO}_{4}$, i.e., $\mathrm{Mg}^{++}$and $\mathrm{SO}_{4}^{--}$ions, which are second to $\mathrm{Na}^{+}$and $\mathrm{Cl}^{-}$in abundance in sea water, does not seem to influence the droplet growth. This indicates strongly that inorganic ions are not responsible for the phenomenon. 4) The effect is reproducible beyond experimental error, can be realistically simulated in the laboratory, and is independent of the actual humidification technique. 5) Because of the similarity in the distribution of crystal sizes from sea water and salt solution, the possibility that the effect is caused by difference in initial sizes of the crystals must be excluded.

The growth of a solution droplet in a humid environment is caused by the vapor pressure gradient between the drop surface and the environment. Combining Raoult's law (which describes the behavior of the vapor pressure of solutions) with the Kelvin relation (which gives the increase of vapor pressure over curved surfaces) results in the well-known relationship between vapor pressure $p_{r}$ of a solution droplet and its size $r$ (Keith and Arons, 1954). Assuming that the larger light scattering for the humidified pure $\mathrm{NaCl}$ aerosol implies that the droplets are larger than those of the sea-water aerosol at a given humidity, some speculation is warranted as to the cause. This assumption is probably valid for the droplets produced in these experiments due to the similar polydispersity (see Fig. 2). Further, since the salt particles grow by a factor of about 1.5-2.0 in radius at the phase change, depending slightly on the initial size of the crystal, the assumption that $b \propto r^{p}$, with $2<p<3$ or perhaps even $p \approx 2$ is justified.

In order for the non-seawater droplets to grow to a larger size, the vapor pressure must be correspondingly smaller or the sea-water drops must be contaminated. For the former case the vapor pressure must be increased at the surface of the sea-water droplets compared to the salt-solution droplets for identically sized droplets at the same temperature. From the parameters influencing the vapor pressure of solution droplets only the somewhat higher gram molecular weight $M$ of sea water compared to pure $\mathrm{NaCl}$ solution could cause the vapor pressure of the sea-water droplet to increase. All

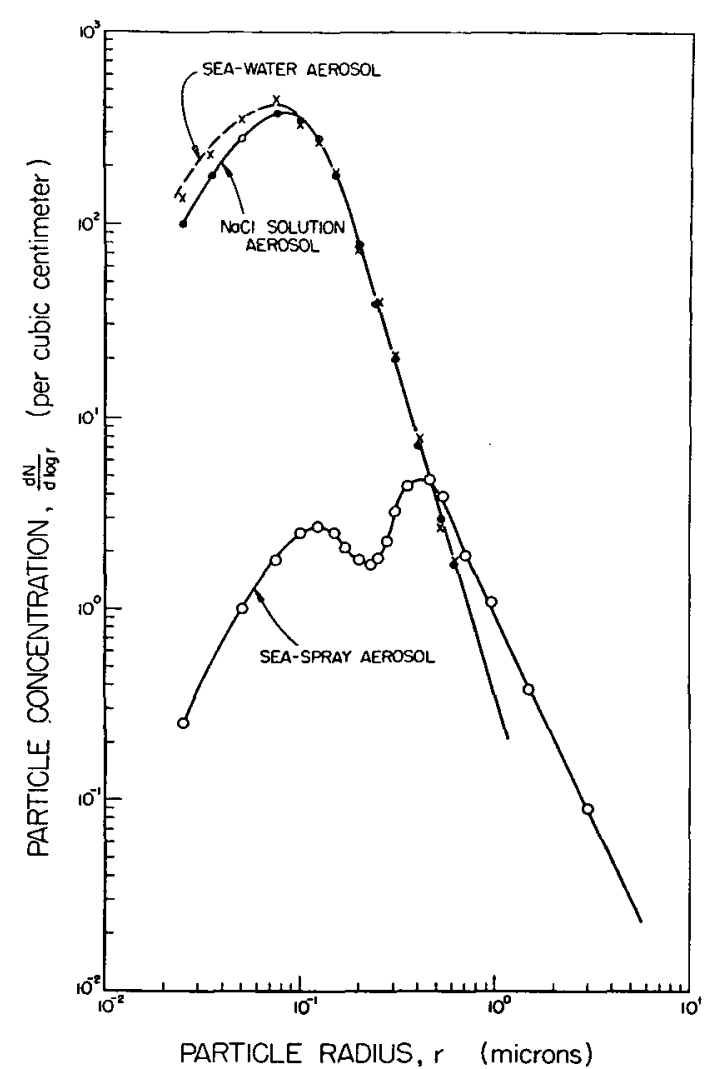

FIG. 2. Particle concentration, $d N / d \log r$ per cubic centimeter as function of particle radius $r$ for a natural sea-spray aerosol and laboratory-produced $\mathrm{NaCl}$ solution and sea water aerosols.

the other variables have the opposite effects, i.e., tend to reduce the vapor pressure of the sea-salt droplet, and a numerical evaluation of these parameters indicates that the combined effects of surface tension, van't Hoff factor, mass of dissolved salt, and density more than counterbalance the effect of $M$.

Deviations from Raoult's law must be anticipated, particularly at relative humidities corresponding to solution droplets of high salt concentration, which is the case shortly after the phase transition has occurred. If the increased vapor pressure of the sea-water droplet is explained in terms of such a deviation, the factors contributing to non-ideality in Raoult's law must cause an increase in the activity of $\mathrm{H}_{2} \mathrm{O}$ and/or a decrease in $\mathrm{Na}^{+}, \mathrm{Mg}^{++}, \mathrm{Cl}^{-}$, etc., ion activity. Dissimilar shapes of molecules, differences in the degrees of freedom of rotation of the molecules, polarity, association of molecules, etc., can cause positive deviations from ideal behavior, i.e., a tendency of separation between the components of the solution. Such a phenomenon,however, is almost the opposite of the basic mechanism of hydration, i.e., a bridging of the ions of $\mathrm{Na}^{+}$and $\mathrm{Cl}^{-}$ by the polar water molecules, which then are bound to the ions by coulombic attractive forces. If, for any reason, these attractive forces are lowered by the presence of foreign substances, more water molecules 
would be "free" to increase the vapor pressure of the droplet. The ocean is known to contain a multitude of organisms which produce a great variety and amount of organic substances. The assumption, therefore, seems justified that some organic molecules with a greater affinity to the $\mathrm{Na}^{+}$and $\mathrm{Cl}^{-}$ions (e.g., a higher dipole moment than that of water) take over the bridging between them, resulting in a greater number of water molecules being available for evaporation. This same mechanism would result in a simultaneous decrease of the van't Hoff factor, an additional effect in the right direction.

An alternate mechanism to explain the growth difference in the two systems might involve the formation of two partially immiscible liquids in the sea-water case, with an organic phase separated from the aqueous phase. This assumption is justified because the critical solution temperature (Glasstone, 1959) of water-organic mixtures is generally above ambient and is raised by the presence of salts which are insoluble in the organic phase. The total vapor pressure of such a system is then constant at a definite temperature irrespective of the relative amount of the two constituents, which follows from the phase rule. Also, in equilibrium, the partial pressures of each component are the same in the two liquid layers. The presence of an organic, more volatile substance could thus increase the vapor pressure of water.

Finally, the possibility of polar organic molecules located at the droplet surface with their hydrophilic functional groups attached to the water and their hydrophobic segments oriented away from the droplet surface, thus changing the hygroscopic nature of the seawater droplet, must be considered. Correlations between atmospheric sea-salt particles and surface-active organic material (Blanchard, 1968) suggest that this substance is carried in to the air in relatively high concentrations by the small droplets that originate at the surface of the sea.

Investigations are underway to explore the mechanism(s) of the effect in order to evaluate its possible contributions to inadvertant weather modification, and to explore to what extent it can be manipulated to intentionally influence the weather.

\section{Summary and conclusion}

It has been shown that the light-scattering coefficient of an aerosol formed by condensing water vapor on sea-spray droplets at $70 \%<R_{T}<90 \%$ relative humidity is significantly less than that of pure $\mathrm{NaCl}$ solution droplets in an identical environment. This effect has been verified reproducibly and beyond systematic and/or random errors of the experiment at the seashore and in the laboratory. Although the mechanism causing the phenomenon is not yet fully understood and still subject to investigation, it is conceivable that organic molecules combine with the $\mathrm{Na}^{+}$and $\mathrm{Cl}^{-}$ions so as to inhibit the attachment of water molecules to these ions. The degrees of freedom of water are thereby increased and the probability for their escape from the liquid enhanced. This results in an increase in vapor pressure above the solution droplets and reduces the size to which the droplet can grow at certain humidities of the environment. A second mechanism of vapor pressure increase can be based on the assumption of formation of partially immiscible liquids of water and some organic substance produced by the ocean. Furthermore, the possibility exists that adsorption of surface-active organic molecules on the droplet surface changes the hydrophilic nature of a solution droplet.

The observed effect could have the following practical implications. 1) Care must be taken in assuming ideal solution properties, or even $\mathrm{NaCl}$ solution properties, in assessing the behavior of natural sea-spray aerosols. 2) At a given relative humidity, visibilities are not as restricted in a maritime environment (Wright, 1939) as would be expected from present knowledge of solution droplet growth [since $L_{v} \propto b^{-1}$ (Horvath and Noll, 1969)]. Differences of up to $70 \%$ can be anticipated at humidities at $\sim 90 \%$. 3) The droplet growth of seawater droplets might not be great enough to make the sea-salt hypothesis an adequate basis for the mechanism of rain formation. 4) If there is a preference of certain molecules to combine with ions in sea-water droplets to significantly change their vapor pressure, it is conceivable that certain man-made water or air pollutants have the same effect so that the problem of inadvertant weather modification has to be considered in this connection.

Because the effect could be caused by man-made activities, and because it apparently has not been observed elsewhere in the world, it could be peculiar to a specific location like the Pacific Northwest. Its possible significance, however, warrants further investigations which it is hoped will be stimulated by this note.

Acknowledgments: The work was supported by Research Grant No. AP - 00336 from the National Air Pollution Control Administration.

\section{REFERENCES}

Ahlquist, N. C., and R. J. Charlson, 1967: A new instrument for evaluating the visual quality of air. $J$. Air Pollution Control A ssoc., 17, 467-469.

Blanchard, D. C., 1968: Surface active organic material on airborne salt particles. Proc. Intern. Conf. Cloud Physics, Toronto, 2.5-29.

Glasstone, S., 1959: Textbook of Physical Chemistry. New York, Van Nostrand, 1320 pp.

Horvath, H., and K. E. Noll, 1969: The relationship between atmospheric light scattering and visibility. Atmos. Environ. (in press).

Keith, C. H., and A. B. Arons, 1954: The growth of sea-salt particles by condensation of atmospheric water vapor. $J$. Meteor., 11, 173-184.

Pueschel, R. F., 1969: Thermal decomposition of sodium containing particles in a flame. $J$. Colloid Interface Sci., 30, 120-127.

Wright, H. L., 1939: Atmospheric opacity: A study of visibility observations in the British Isles. Quart. J. Roy. Meteor. Soc., $65,411-439$. 